\title{
Effects of Water Activity and Culture Age on the Glycerol Accumulation Patterns of Five Fungi
}

\author{
By AILSA D. HOCKING \\ CSIRO Division of Food Research, North Ryde, NSW 2113, Australia
}

(Received 20 March 1985; revised 7 June 1985)

\begin{abstract}
One non-xerophilic fungus, Penicillium digitatum, and four xerophilic fungi, Penicillium janczewskii, Eurotium chevalieri. Wallemia sebi and Polypaecilum pisce, were grown at six different water activities $\left(a_{n}\right)$ on media containing various concentrations of sodium chloride. Each species was sampled as soon as visible growth appeared and up to six times thereafter during various stages of the growth cycle. The fungal mycelium was extracted and assayed for glycerol using a specific enzymic method. At the highest $a_{w}, 0.997$, only small amounts of glycerol were present in the fungi. At lower $a_{w}$ values, glycerol concentrations rose rapidly at first, then declined as the cultures aged. There appeared to be a correlation between the amount of glycerol accumulated, and the complexity of the spore-bearing structures. Glycerol depletion appeared to be related to the formation of spores and their maturation.
\end{abstract}

\section{INTRODUCTION}

In a study on the osmoregulatory solutes of five fungi, Hocking \& Norton (1983) reported that glycerol was the major internal solute in four xerophilic fungi and one non-xerophilic species. All fungi tested were able to accumulate glycerol when grown on media of low water activity $\left(a_{\mathrm{w}}\right)$ with glucose/fructose or sorbitol added to adjust the $a_{\mathrm{w}}$. However, when sodium chloride was used as the controlling solute in the growth media, only one of the five species tested, Wallemia sebi, was shown to accumulate glycerol below $0.92 a_{w}$, even though two of the other species studied, Penicillium janczewskii and Eurotium checalieri, were capable of growth on salt-based media down to $0.85 a_{\mathrm{w}}$, the lowest $a_{\mathrm{w}}$ tested. Hocking \& Norton (1983) suggested that perhaps these fungi were using an alternative. non-organic osmoregulatory solute such as potassium. Alternatively, since the cultures were incubated for several weeks to produce sufficient material for analysis by natural abundance ${ }^{13} \mathrm{C}$ NMR, it was possible that much of the mycelium was senescent and had lost or metabolized the accumulated glycerol.

This study was designed to investigate this latter hypothesis by examining the time-course of glycerol accumulation in four of the fungi previously studied by Hocking $\&$ Norton (1983) and a fifth species recently described from salted fish by Pitt \& Hocking (1985).

\section{METHODS}

Fungi. Four xerophilic fungi were studied, Penicillium janczewskii Zaleski FRR1618, Wallemia sebi(Fries) v. Arx FRR 1471, Eurotium chevalieri Mangin FRR 547 and Polypacilum pisce Hocking \& Pitt FRR2788, together with one non-xerophilic species, Penicillium digitatum (Pers.: Fr.) Sacc. FRR 1562. Poly. pisce was recently described by Pitt \& Hocking (1985) as the dominant spoilage fungus on dried and salted fish from Indonesia. Xeromyces hisporus Fraser was omitted from the present experiments because it grows poorly or not at all on salt-based media (Pitt $\&$ Hocking, 1977; Hocking \& Norton, 1983).

Media. The basal medium used for the glycerol accumulation studies was $(\mathrm{w} / \mathrm{v}): 1 \%$ yeast extract: $5 \%$ glucose; $0.4{ }_{0}^{\circ} \mathrm{KH}_{2} \mathrm{PO}_{4} ; 2 \%$ agar; $\mathrm{pH} 6 \cdot 2$. This basal medium was supplemented with $\mathrm{NaCl}$ to produce media of six different water activities reflecting the growth ranges of the fungi studied. The appropriate $\mathrm{NaCl}$ concentrations were calculated from the data of Robinson \& Stokes (1955). For the four xerophilic fungi, the six media were of $0.997,0.99,0.96,0.92,0.88$ and $0.85 a_{n}$. The $a_{n}$ of these media, measured using a Sina-Scope instrument 
(Novasina, Zurich, Switzerland), did not devia e from the calculated $a_{n}$ by more than $\left.0 \cdot 0\right) a_{w}$ units. For $P$. digitatum, which is unable to grow below $0.92 a_{n}$ in NaCl-based media (Hocking \& Pitt. 1979), the stx medial were of $0.997,0.99,0.975,0.96,0.945$ and $0.93 a_{n}$

Cutriwation and sampling. Glass Petri dishes, $140 \mathrm{~mm}$ in diameter, containing $50 \mathrm{ml}$ of medium were overlaid with a circle of dialysis membrane (Union Carbide). Six Petri dishes were prepared for each species at each $a_{w}$. The plates were inoculated with spores suspended in distilled water containing $0.05 \%(\mathrm{v} / \mathrm{v})$ Tween 80 . Approximately $0.1 \mathrm{ml}$ of suspension was pipetted onto the membrane and spread with a sterile glass spreader. The Petri dishes were then sealed with adhesive cellulose tape, placed in polyethylene bags to minimize moisture loss and incubated in the upright position at $25^{\circ} \mathrm{C}$. Sealing the Petri dishes in this way did not appear to adversely affect growth, since polyethylene is highly permeitble to oxygen, and the gruwing cultures sporulated readily and showed no signs of oxygen limitation. Samples were taken at intervals according to the grow th rate of each fungus at each $a_{n}$, so that each species was exammed soon after germination (microcolony stage), during spore formation and maturation, and into senescence. Before removing the fungal mycelium for glycerol extraction, a small portion was examined microscopically to determine the pesence and maturity of conidia or ascospores, and the degree of degeneration (If any) of the hyphae. The presence of granulation and lipid globules inside the hyphac or free in the mounting medium were taken to be signs of degeneration and aging.

Glycerol extraction. The mycelium from an ertire Petri dish was used at each sampling time. The dialysis membrane was lifted from the agar and the total imount of mycelial growth was removed and weighed. In some instances where there was sufficient mycelial growth at the low $a_{n}$ values, the last Petri dish in the series was sampled twice, using only half the mycelium at each sampling time, thus adding an extra data point wo the glycerol accumulation curve. Glycerol was extracted using the method of Maclean \& Scott (1976) as modified by Hocking \& Norton (1983). Weighed mycelium was translerred to a flask containing $30 \mathrm{ml} 80^{\circ}$ ( $(\mathrm{v} / \mathrm{v})$ aqueous ethanol, which was then heated to boiling in a water bath and bouled vigorously for $10 \mathrm{~min}$. The mycelium was removed by filtration through a Buchner tunnel on Whatman no. I filter paper. The extract was evaporated to dryness under vacuum at $55^{\circ} \mathrm{C}$ in a rotary evaporator. redisso ved in a known volume of distilled water, and then stored at $-18 \mathrm{C}$ until analysed.

After removal of the fungal growth on the dialysis membrane, three agar blocks totalling $10 \mathrm{~g}$, were cut from the spent growth medium. The blocks were transferred to a plastic bag containing $100 \mathrm{ml} 80^{\circ}{ }_{0}$. atqueous ethanol and homogenized in a Coleworth Stomacher (Seward, Bury St Fdmunds, UK) for 2 mm. The homogenate was decanted onto a Buchner funnel and the agar rernoved by filtration through two layers of Whatman no. 1 filter paper. The agar was then re-extracted by repeating the process with a further $50 \mathrm{ml} 80^{\circ}$, acpueous ethanol. The filtrates were combined, reduced in volume and stored as for the mycelial extracts. Experiments using agar containing known concentrations of glycerol showed that this procedure extracled approximately $99^{\circ}$ of the glycerol: $90^{\circ}$, with the first extraction, and $90^{\circ}$ of the remainder with the second extraction

Glycerol analysis. Glycerol was determined using a commercially available en Mannheim glycerol test combination, catalogue no, 148270) based on the method of Fggstein \& Kuhlmann (1974) The method was scaled down to one-quarter volume and the reactions and analyses were done in $1 \cdot() \mathrm{ml}$ quartz spectrophotometer cells. The scaling down did not affect the sensitivity of the method. Fxperiments showed that the enzyme system was highly specific for glycerol and was unaffected by erythritol, arabitol, mannitol, sorbitol or glucose, even when these compounds were present in concentrations ten times that of glycerol.

\section{RESULTS}

The patterns of glycerol accumulation in the five fungi studied are shown in Figs 1 to 5. All five species showed a similar basic pattern, although there were some variations which will be discussed later. As the $a_{n}$ decreased, the maximum amount of glycerol accumulated increased, and the time-course of the accumulation and depletion became longer. In most cases, the commencement of production of mature (free) conidia occurred at or near the peak of glycerol accumulation (see Table 1 and Figs 1 to 5) and sporulation was followed by a dramatic fall in the intracellular glycerol concentration. This drop in glycerol appeared to accompany the degeneration of both the vegetative mycelium and the spore-bearing structures.

$P$. digitatum (Fig. 1) was able to accumulate significant quantities of glycerol, even at the lowest $a_{\mathrm{w}}(0.93)$ at which it was grown, and even though it is not a xerophile. However, it appeared to lack mechanisms for retaining high intracellular concentrations of glycerol at reduced $a_{w}$, since considerable amounts of glycerol were detected in the growth media (Table 2). The maximum amount of glycerol accumulated at $0.93 a_{\mathrm{w}}$ was less than the maximum at $0.945 a_{\mathrm{w}}$, perhaps an indication that at $0.93 a_{\mathrm{w}}$ this species was close to the limit of its $a_{\mathrm{w}}$ growth range. 


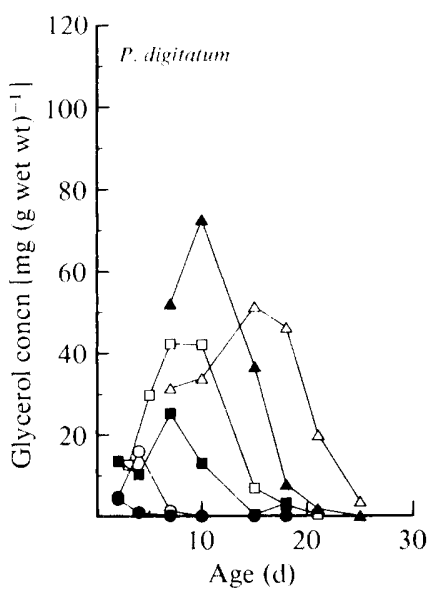

Fig. I

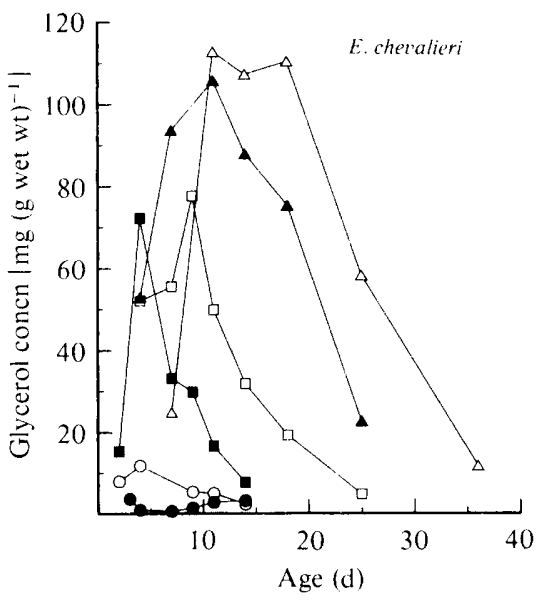

Fig. 2

Fig. 1. Effect of $a_{n}$ and culture age on the internal glycerol concentration of $P$. digitatum., $0.997 a_{4}$; $0.0 .99 a_{w}: \square .0 .975 a_{n}: \square, 0.96 a_{n} ; \mathbf{\Delta}, 0.945 a_{n} ; \triangle, 0.93 a_{n}$.

Fig. 2. Fffect of $a_{w}$ and culture age on the internal glycerol concentration of E. cheralieri. $0.997 a_{w}$; $0,0.99 a_{n}: \boldsymbol{\square}, 0.96 a_{n}: \square .0 .92 a_{\mathrm{w}} ; \mathbf{\Delta}, 0.88 a_{\mathrm{w}}: \triangle, 0.85 a_{\mathrm{w}}$.

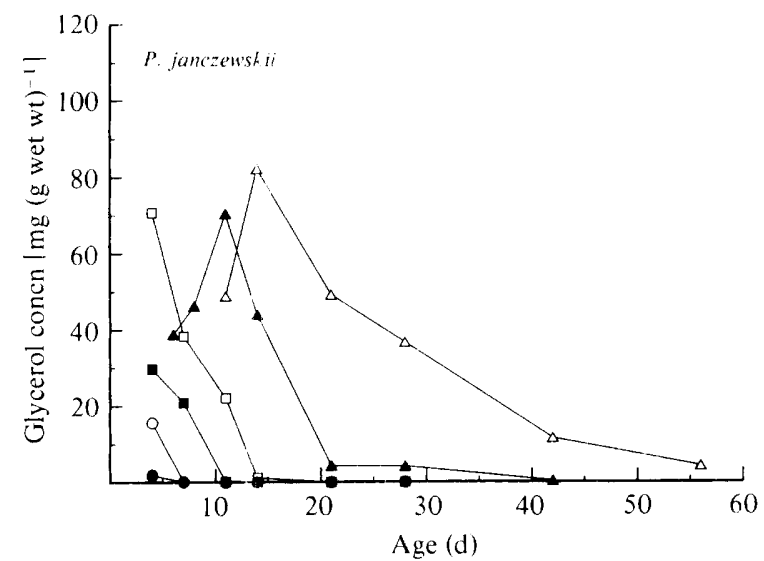

Fig. 3. Effect of $a_{n}$ and culture age on the internal glycerol concentration of $P$. janczewskii. $0,0.997 a_{n}$; $0,0.99 a_{n}: 0,06 a_{n}: \square, 0.92 a_{n}: \mathbf{\Lambda}, 0.88 a_{n} ; \triangle, 0.85 a_{n}$.

The glycerol accumulation patterns of the four xerophilic species showed some interesting differences. E. cheralieri (Fig. 2), which as an ascomycete has a more complex sporulation cycle than the other three xerophiles, produced much more glycerol, especially at lower $a_{\mathrm{w}}$ values $(0 \cdot 88$ to 0.85). At $0.92 a_{\mathrm{w}}, E$. chevalieri produced free ascospores within $14 \mathrm{~d}$ (Table 1). At 0.88 and $0.85 a_{\mathrm{w}}$, although the peak concentration of glycerol was higher, and it was retained much longer, no mature ascospores were produced at either $a_{\mathrm{w}}$ within the time-span of the experiment ( $25 \mathrm{~d}$ at $0.88 a_{\mathrm{w}}$ and $36 \mathrm{~d}$ at $0.85 a_{\mathrm{w}}$ ). Had the experiment been continued over a longer period, ascospores may eventually have been produced.

$P$. janczewskii (Fig. 3) responded to reduced $a_{w}$ by accumulating increasing quantities of glycerol and, at the lower $a_{\mathrm{w}}$ values, by retaining it for longer. At each $a_{\mathrm{w}}$ the appearance of free conidia in the microscopic preparation corresponded with the peak of glycerol accumulation (see Table 1). The onset of sporulation was followed by a rapid loss of glycerol from the mycelium except at the lowest $a_{\mathrm{w}}(0 \cdot 85)$, where the loss was much more gradual. 


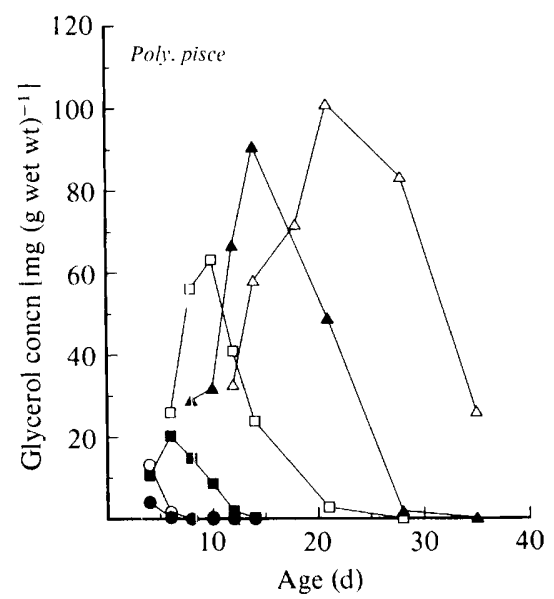

Fig. 4. Effect of $a_{n}$ and culture age on the nternal glycerol concentration of Poly pisce. O. $0.99 a_{u} ; \boldsymbol{\square}, 0.96 a_{\mathrm{w}} ; \square, 0.92 a_{w} ; \boldsymbol{\Delta}, 088 a_{\mathrm{w}} ; \triangle, 0.85 a_{\mathrm{w}}$

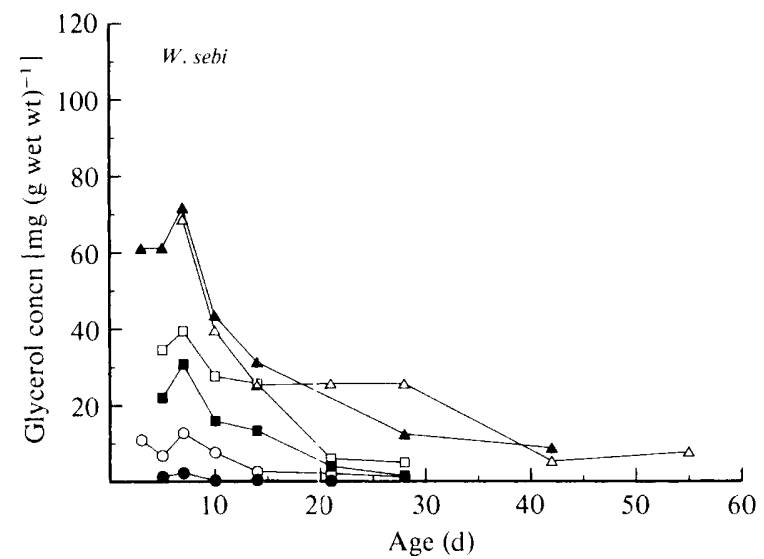

Fig. 5. Fffect of $a_{n}$ and culture age on the internal glycerol concentration of $W$. sebi. $0,0.99 a_{n}: \boldsymbol{\square}, 0.96 a_{n}: \square, 0.92 a_{n}: \mathbf{\Delta}, 0.88 a_{k}: \triangle, 0.85 a_{w}$.

Table 1. Sporulation time (d) for fungi grown on salt-based media

\begin{tabular}{|c|c|c|c|c|c|c|c|}
\hline Species & $a_{\mathrm{n}}$ & 0.997 & 0.99 & 0.96 & 0.92 & 0.88 & $0 \cdot 85$ \\
\hline P. digitatum & & $1-2$ & 2 & 5 & & & \\
\hline P. janczewskii & & 34 & 3 & 3 & $4-5$ & 1011 & 14 \\
\hline $\begin{array}{l}\text { E. cherallert } \\
\text { Conidia }\end{array}$ & & $3-4$ & 3 & 34 & 4 & 7 & $10-11$ \\
\hline Ascospores & & 11 & 10 & 11 & 14 & $(25)^{*}$ & $(36)^{*}$ \\
\hline Poly pisce & & 6 & 4 & 4 & 6 & $11 \cdots 12$ & 1617 \\
\hline W. sebi & & 12 & 1 & 1 & 2 & 3 & 5 \\
\hline
\end{tabular}

* No cleistothecia or ascospores produced by the time the last sample was taken.

The pattern for Poly. pisce (Fig. 4) was similar to that for P. janczewskii. The highest concentration of glycerol was recorded at the lowest $a_{\mathrm{w}}$. At lower $a_{\mathrm{w}}$ values, the peak of glycerol accumulation occurred after the observation of the first mature conidia (Table 1), but coincided with the period of maximum sporulation. No degeneration of vegetative mycelium or sporebearing structures (conidiophores) was noted at the peak glycerol concentration, but aging was 
Table 2. Concentration of glycerol in the growth media of three fungi corresponding with (a) the peak of glycerol accumulation and (b) glycerol depletion in the mycelium

\begin{tabular}{|c|c|c|c|c|c|}
\hline \multirow[b]{3}{*}{ Wallemia sebi } & \multirow{3}{*}{$\begin{array}{c}a_{\mathrm{u}} \\
0.997\end{array}$} & \multicolumn{4}{|c|}{ Glycerol concn $\left(\mathrm{mg} \mathrm{g}^{-1}\right)$} \\
\hline & & \multicolumn{2}{|c|}{ (a) } & \multicolumn{2}{|c|}{ (b) } \\
\hline & & $7 \mathrm{~d}$ & 0.78 & $14 \mathrm{~d}$ & $0 \cdot 02$ \\
\hline & 0.99 & $7 \mathrm{~d}$ & 3.92 & $28 \mathrm{~d}$ & $0 \cdot 86$ \\
\hline & 0.96 & $7 \mathrm{~d}$ & $5 \cdot 04$ & $28 \mathrm{~d}$ & 3.94 \\
\hline & 0.92 & $7 \mathrm{~d}$ & 1.53 & $28 \mathrm{~d}$ & $2 \cdot 40$ \\
\hline & $0 \cdot 88$ & $7 \mathrm{~d}$ & 0.09 & $42 \mathrm{~d}$ & 4.09 \\
\hline & $0 \cdot 85$ & $10 \mathrm{~d}$ & $1 \cdot 04$ & $55 \mathrm{~d}$ & 1.85 \\
\hline \multirow[t]{6}{*}{ Penicillium janczewskii } & 0.997 & $4 d$ & $0 \cdot 15$ & $14 d$ & $0 \cdot 01$ \\
\hline & 0.99 & $4 \mathrm{~d}$ & $0 \cdot 13$ & $14 \mathrm{~d}$ & 0.01 \\
\hline & 0.96 & $4 \mathrm{~d}$ & $0 \cdot 11$ & $14 \mathrm{~d}$ & 0 \\
\hline & 0.92 & $4 \mathrm{~d}$ & 0.06 & $21 \mathrm{~d}$ & 0 \\
\hline & $0 \cdot 88$ & $11 \mathrm{~d}$ & $0 \cdot 01$ & $28 \mathrm{~d}$ & $0 \cdot 05$ \\
\hline & $0 \cdot 85$ & $14 \mathrm{~d}$ & $0 \cdot 14$ & $28 \mathrm{~d}$ & $0 \cdot 05$ \\
\hline \multirow[t]{6}{*}{ Penicillium digitatum } & 0.997 & $2 \mathrm{~d}$ & $0 \cdot 18$ & $10 \mathrm{~d}$ & 0 \\
\hline & 0.99 & $4 d$ & 0.66 & $10 \mathrm{~d}$ & 0 \\
\hline & 0.975 & $7 d$ & 1.05 & $15 \mathrm{~d}$ & 0 \\
\hline & 0.96 & $7 d$ & $4 \cdot 55$ & $21 \mathrm{~d}$ & $0 \cdot 02$ \\
\hline & 0.945 & $10 \mathrm{~d}$ & 5.80 & $25 \mathrm{~d}$ & 0 \\
\hline & 0.93 & $15 \mathrm{~d}$ & 8.41 & $25 \mathrm{~d}$ & 0.01 \\
\hline
\end{tabular}

usually evident in the first sample on the downward side of the glycerol curve. At $0.85 a_{\mathrm{w}}$, in contrast to $P$. janczewskii, glycerol was lost by Poly. pisce at a rate similar to that at the higher $a_{\mathrm{w}}$ values.

For $W$. sebi (Fig. 5), the glycerol accumulation and depletion patterns showed some significant differences from those of the other xerophilic fungi. The highest glycerol concentration was recorded at $0.88 a_{\mathrm{w}}$, rather than $0.85 a_{\mathrm{w}}$.W. sebi accumulated less glycerol than the other fungi studied, but the intracellular glycerol was lost at a much slower rate. At the lower $a_{n}$ values $(0.92$ to 0.85$)$ a significant amount of glycerol remained in the conidia and mycelium after $42 \mathrm{~d}\left(0.92\right.$ and $\left.0.88 a_{\mathrm{w}}\right)$ and $55 \mathrm{~d}\left(0.85 a_{\mathrm{w}}\right)$, indicating either that some vegetative mycelium was still functioning, or that the conidia themselves contained some glycerol.

Some glycerol was lost to the growth media (Table 2), particularly when the concentration of glycerol in the fungal mycelium was highest. Glycerol was detectable in the growth media of $W$. sebi long after sporulation had first been observed, and much of the mycelium had degenerated, especially at the lower $a_{u}$ values. P. janczewskii was much more conservative and lost very little of its glycerol to the growth media, even at the peak mycelial glycerol concentration at the lowest $a_{\mathrm{w}} . P$. digitatum lacked this mechanism for conserving glycerol. With each decrease in $a_{\mathrm{w}}$, the amount of glycerol detectable in the growth media increased, at the time when the mycelial glycerol was highest. However, little or no glycerol was detectable in the growth media at any $a_{w}$ when the glycerol in the mycelium of $P$. digitatum was depleted.

\section{DISCUSSION}

The results presented above explain the apparent drop in internal glycerol concentration observed by Hocking \& Norton (1983) for $P$. janczewskii and $E$. chevalieri grown at $0.85 a_{n}$ on salt-based media. In that study, cultures were several weeks old when sampled at the lowest $a_{\mathrm{w}}$, but were much younger at medium and high $a_{\mathrm{w}}$ values. It would appear from the results presented here that, in the study of internal osmoregulatory solutes in filamentous fungi, consideration must be given to the culture age when sampling, as glycerol is lost as cultures senesce.

Luard (1982) reported that glycerol was the principal osmoregulatory solute accumulated by Penicillium chrysogenum when it was grown on $\mathrm{KCl}$-based media over a range of osmotic potentials from 0 to $-10 \mathrm{MPa}$, the latter being equivalent to $0.93 a_{\mathrm{w}}$ (see formula in Luard \& 
Griffin, 1981). Luard (1982) observed a steady rise in the internal glycerol content of $P$. chry'sogenum in response to reduction in the $a_{\mathrm{w}}$ of the growth medium, but did not report the age at which the fungus was sampled at each osmotic potential. Samples for analysis were taken when the radius of the colony, grown from a $4 \mathrm{~mm}$ agar plug, was at least $20 \mathrm{~mm}$. The age of such a colony can be calculated from the data on the growth rates of $P$. chrysogenum published by Luard \& Griffin (1981). At $-10 \mathrm{MPa}, P$. chrysogenum would reach a colony diameter of $20 \mathrm{~mm}$ in 8 to $9 \mathrm{~d}$, at $-6 \mathrm{MPa}\left(0.955 a_{\mathrm{w}}\right)$ in 7 to $8 \mathrm{~d}$, and at $-4 \mathrm{MPa}\left(0.97 a_{\mathrm{w}}\right)$ in 5 to $6 \mathrm{~d}$. Since the water relations of $P$. chrysogenum are similar to those of $P$. janczewskii (Hocking $\&$ Pitt, 1979), it can be assumed that the glycerol accumulation patterns of these two species would be similar. Figure 3 shows that, at the ages calculated for he sampling of $P$. chrysogenum, Luard (1982) would probably have been sampling slightly on the downward side of the glycerol accumulation curve at each $a_{\mathrm{w}}$. Since each sample at each $a_{\mathrm{w}}$ was taken at approximately the same stage of the glycerol accumulation curve, Luard (1982) was able to demonstrate the expected relationship between lower $a_{\mathrm{w}}$ and higher glycerol concentration.

What happens to the glycerol that is 'lost' from the mycelium? Analyses of the growth media (Table 2) showed that some glycerol was lost to the medium when the intracellular glycerol concentration in the mycelium was at its highest. However, this glycerol must subsequently have been metabolized by the fungi, as little or no detectable glycerol remained in the growth medium when the intracellular glycerol was exhausted. W. sebi was an exception, as some glycerol remained in the growth media, particularly at the lower $a_{\mathrm{u}}$ values. However, at the same time, glycerol was still present in the hyphae of this species in higher concentrations than in the media.

The data presented here show a good correlation between glycerol accumulation and the production of mature conidia (Table 1, Figs 1 to 5). Intracellular glycerol began to decline only after a reasonable proportion of the mycelium had sporulated. This information sheds little light, however, on the basic question of how some fungi are able to grow at low $a_{\mathrm{w}}$, and others not. Although the glycerol accumulation pattern for the non-xerophilic species $P$. digitatum was similar to that for $P$. janczewskii, the $a_{\mathrm{w}}$ range for growth was more restricted. $P$. digitatum is obviously capable of producing glycerol down to $0.945 a_{\mathrm{w}}$, but below that $a_{\mathrm{w}}$, glycerol production slows, and growth ceases at about $0.92 a_{w}$ in salt-based media, and at about $0.90 a_{w}$ in carbohydrate-based media (Hocking \& Pitt, 1979). Table 2 shows that $P$. digitatum is unable to prevent glycerol leakage from its hyphae, as the amount of glycerol present in the growth media increased as the $a_{\mathrm{u}}$ decreased. This inability to retain glycerol is perhaps the reason why $P$. digitatum is unable to grow at low $a_{\mathrm{w}}$. The comparison between $P$. janczewskii and $P$. digitatum is interesting: $P$. janczewskii, a xerophile, is able to retain its intracellular glycerol against a strong concentration gradient, whereas $P$. digiratum, a non-xerophile, leaks glycerol to the growth medium. A parallel situation was described by Brown (1978) for the xerophilic yeast Saccharomyces rouxii and the closely related but non-xerophilic species, Saccharomyces cerevisiae.

The data provide some information about the success of $W$. sebi as a spoilage fungus in commodities containing a high proportion of salt (Frank \& Hess, 1941). Of the five fungi studied, $W$. sebi produces the smallest conidia on the simplest conidiophores, and it produces them very rapidly and in vast quantities. At 0.997 to $0.92 a_{\mathrm{w}}$, within $24 \mathrm{~h}$ of germination (and often within $24 \mathrm{~h}$ of inoculation), when growth was barely visible macroscopically, microscopic examination revealed numerous conidiophores and free conidia. Even at the lowest $a_{\mathrm{w}}$ tested, $0.85, W$. sebi had produced spores within $5 \mathrm{~d}$ of inoculation (Table 1) which is within $3 \mathrm{~d}$ of germination. This sporulation time at $0.85 a_{\mathrm{w}}$ is rapid compared with the other fungi studied: $P$. janczewskii, $14 \mathrm{~d}$ from inoculation; Poly. pisce, 16 to $17 \mathrm{~d}$ and E. chevalieri 10 to $11 \mathrm{~d}$ (for conidia only). The process by which $W$. sebi produces its conidia may well require far less energy than the production of the more complex conidiophores of Penicillium, Polypaecilum and Aspergillus. The production of ascospores (e.g. in Eurotium species) is an even slower process which probably requires more energy than the production of conidia. Eurotium species cease to produce their teleomorphic state at an $a_{\mathrm{w}}$ significantly higher than their minimum $a_{\mathrm{w}}$ for growth (Pitt \& Christian, 1968; Curran, 1971). E. chevalieri may be incapable of producing ascospores below 
about $0.92 a_{\mathrm{w}}$ in salt-based media, as the teleomorph is not produced below $0.843 a_{\mathrm{w}}$ under favourable conditions on media containing high concentrations of carbohydrate (Pitt \& Christian, 1968). W. sebi retained its intracellular glycerol much longer than the other four species investigated. Microscopic examination showed that $W$. sebi cultures contained active conidiophores for a considerable time after sporulation was first noted. The cultures of this species consisted of a thin mycelial mat which was quickly covered with a crust of brown conidia. The ratio of conidial to mycelial mass would have been much higher for $W$. sebi than for any of the other species included in this study.

An explanation of the greater success of some xerophilic fungi in high salt environments may be gained by an investigation of the energy requirements for reproduction and maintenance in salt tolerant and non-tolerant species. It may be that the maintenance energy requirements of fastidious xerophiles such as Xeromyces bisporus and Chrysosporium fastidium are high, restricting such fungi to energy-rich environments like dried fruits, confectionery etc. Neither of these species grows well in media containing high concentrations of salt (Pitt \& Hocking, 1977; Luard \& Griffin, 1981). The inability of these and other species to grow on salt-based media may also be due to an intolerance of salt, and a lack of mechanisms to deal with high concentrations of sodium and similar positively charged ions. Many of the differences between the responses of the four xerophilic fungi studied here may not have been so apparent if they had been grown on energy-rich media based on glucose or some other sugar.

\section{REFERENCES}

Brown, A. D. (1978). Compatible solutes and extreme water stress in eukaryotic micro-organisms. $A d$ rances in Microbial Physiology 17, 181-242.

Curran, P. M. T. (1971). Sporulation in some members of the Aspergillus glaucus group in response to osmotic pressure, illumination and temperature. Transactions of the British Mycological Society 57, 20) -211

Eggstein, M. \& Kuhlmann, E. (1974). Triglycerides and glycerol. Determination after alkaline hydrolysis. In Methods of Enzymatic Analysis. pp. 1825 1831. Edited by H. U. Bergmeyer. London: Academic Press.

Frank, M. \& Hess, E. (1941). Studies on salt fish. VI. Halophilic brown molds of the genus Sporendonema emend. Ciferri et Redaelli. Journal of the Fisheries Research Board of Canada 5, 287-292

HoCkING, A. D. \& NORTON, R. S. (1983). Naturalabundance ${ }^{1.3} \mathrm{C}$ nuclear magnetic resonance studies on the internal solutes of xerophilic fungi. Journal of General Microbiology 129, 2915-2925.

Hocking, A. D. \& PITT, J. I. (1979). Water relations of some Penicillium species at $25^{\circ} \mathrm{C}$. Transactions of the British Mycological Society 73, 141-145.
LUARD, E. J. (1982). Accumulation of intracellular solutes by two filamentous fungi in response to growth at low steady state osmotic potential. Journal of General Microbiology 128, 2563-2574.

LUARD, E. J. \& Griffin, D. M. (1981). Effect of water potential on fungal growth and turgor. Transactions of the British Mycological Society 76, 33-40.

MAClEAN, D. J.\& SCOTT, K. J. (1976). Identification of glucitol (sorbitol) and ribitol in a rust fungus Puccinia graminis f. sp. tritici. Journal of General Microbiology 97, 83-89

PitT, J. I. \& Christian, J. H. B. (1968). Water relations of xerophilic fungi isolated from prunes. Applied Microbiology 16, 1853-1858.

PITT, J. I. \& Hocking, A. D. (1977). Influence of solute and hydrogen ion concentration on the water relations of some xerophilic fungi. Journal of General Microbiology 101, 35-40.

PitT, J. I. \& Hocking, A. D. (1985). New species of fungi from Indonesian dried fish. Mycotaxon 22, 197-208.

Robinson, R. A. \& Stokes, R. H. (1955). Electrolyte Solutions. London: Butterworth. 